\title{
Ethnic Markers and How to Find Them
}

\section{An Ethnographic Investigation of Marker Presence, Recognition, and Social Information}

\section{Adrian Viliami Bell ${ }^{1}$ (D) Alina Paegle ${ }^{1}$}

Accepted: 4 May 2021 / Published online: 9 June 2021

(c) The Author(s), under exclusive licence to Springer Science+Business Media, LLC, part of Springer Nature 2021

\begin{abstract}
Ethnic markers are a prominent organizing feature of human society when individuals engage in significant anonymous interactions. However, identifying markers in natural settings is nontrivial. Although ad hoc assignment of markers to groups is widely documented in the ethnographic literature, predicting the membership of individuals based on stylistic variation is less clear. We argue that a more systematic approach is required to satisfy the basic assumptions made in ethnic marker theory. To this end we introduce a three-step ethnographic method to assess the presence, recognition, and transmission of markers of group identity: (1) continual scans, (2) a utilization survey, and (3) a comparative classification task. Applying the method to a study of culturally significant motifs in the South Pacific Island nation of Tonga, we provide evidence that the motif set satisfies basic theoretical assumptions and thus the motifs are likely expressions for social coordination. We also found that the coordinating role of each motif is variable and requires further investigation.
\end{abstract}

Keywords Coordination - Ethnic markers $\cdot$ Signals $\cdot$ Cultural evolution $\cdot$ Kupesi . Tonga

The prevalence of cooperation and social coordination depends on the structure of interactions between individuals, with greater benefits accruing to those who assort with others with similar social norms or behaviors (Axelrod \& Hamilton, 1981; Hamilton, 1964). As a result, the ability to recognize features of others is assumed to be under selection (Jansen \& Baalen, 2006; Riolo et al., 2001; Traulsen \& Nowak, 2007). Although individual recognition or somatic features might play an assortative

Adrian Viliami Bell

adrian.bell@anthro.utah.edu

1 Department of Anthropology, University of Utah, 260 S. Central Campus Drive Rm. 4625, Salt Lake City, UT 84112, USA 
role in some contexts (McPherson et al., 2001), in larger, more demographically complex populations, somatic features have strong limitations for predicting the behavior or norms of others (Wimmer \& Lewis, 2010). For example, modern-day return migrants such as the Aussiedler of Germany and nikkeijin of Japan are treated as foreign immigrants despite sharing a genetic ancestry with their hosts (Tsuda, 2009). Even among close consanguineous kin, shared norms or traits are not guaranteed. In such cases other, more flexible features may be the basis of recognition and boundaries between groups with shared traits.

It is in larger, multi-ethnic populations where ethnic marker theory becomes an important explanatory framework for how individuals coordinate their norms and behaviors with others (Boyd \& Richerson, 1987; McElreath et al., 2003). To work, these ethnic markers, sometimes called "identity signals" or "tags," are required to be observable and signify group membership. Markers are considered arbitrary in the sense that they are not required to be costly, such that any sartorial (Wiessner, 1984) or linguistic (Cohen, 2012; Cohen \& Haun, 2013) marker may evolve along cultural and linguistic phylogenetic lines. The finer details of the theory prescribe that markers become more pronounced where more distinct groups are present (McElreath et al., 2003).

Although predictions from ethnic marker theory have received experimental support (Efferson et al., 2008) and ethnographic assertions of marking are plentiful, empirically verifying objects as markers and thus active centers of social coordination has proven much more difficult. The literature reports both a poor and a good mapping of stylistic variation on groups. Among Kalahari San hunters, Wiessner (1983) reports that although interviewed !Kung hunters $(N=55)$ all self-reported that they could identify their own arrows, only two of the ten who were tested could correctly identify their arrows out of a wider set. At the level of the language group, where greater material variation was found, a sample $(N=16)$ of San informants reacted strongly to the arrows of other linguistic groups. However, Wiessner points out that no single arrow feature consistently marked linguistic group affiliation.

In work among pottery producers in the Ecuadorian Amazon, Bowser (2000) found that on average $68 \%$ and $62 \%$ of women correctly identified the political or ethnic group affiliation of the pottery maker, respectively, based on a pottery sample. As in the Wiessner study, Bowser found variable roles of features and combinations likely at play. Other foundational studies report a poorer mapping of stylistic variation group membership (Hodder, 1977; Wiessner, 1984). In more recent work, a vignette study showed high variability in supported traits between age classes in a contemporary urban and agropastoral population (Moya \& Boyd, 2016). These studies reflect the state of the literature: that the link between objects and group identification is highly varied, and that greater analytical measures are required to directly assess what objects, if any, are acting as markers.

We argue that identifying markers is difficult without empirically addressing the basic assumptions of the mechanistic theory. The models by McElreath et al. (2003) and Boyd and Richerson (1987) are primary formulations. In the model by McElreath et al. (2003), a two-person coordination game is played with a marginal benefit to coordinating on the same behavior. Successful coordination gives greater benefits to actors who either randomly assort or have a bias to interact with those with the same marker. 
Actors with higher payoffs transmit their phenotype-both behavior and marker-with greater frequency. The key result from this model is that a covariance develops between behaviors and markers under reasonable conditions, showing that honest signals of behavior, and (ethnic) group affiliation, can evolve.

In a two-patch model by Boyd and Richerson (1987), a social learning strategy was explored where actors adopted the higher-payoff behaviors among individuals with more similar markers. The patches had different optimal behaviors and actors could move between patches, carrying with them their behavior and marker from the previous patch. The model demonstrates that patch-specific optimal behaviors can be maintained with this social learning strategy that combines assortment along markers and success-biased social learning. This result highlights the role markers can play in aiding individuals as they navigate complex heterogeneous environments.

Some features of the markers in these two models are important to an empirical investigation. The signals had no explicit cost. However, it was requisite that markers were transmitted with some accuracy. This allows arbitrary markers to hold information and, by extension, opens the door for individuals to be selective about interactions based on markers. Individuals that do not use markers would be at a disadvantage, and selection would favor the individual to recognize displayed markers and the information they contain.

There are three nontrivial features of the above italicized statement. The markers (1) are present and observable in the population, (2) are widely recognized by individuals, and (3) have information content. In our view, these three features are ethnographic efforts on their own. To this end, this paper presents a systematic ethnographic approach to identify a set of candidate markers and infer their presence to be the result of social coordination. Using scans, an ethnographic survey, and a classification task, we address features of the models and infer whether a set of culturally salient objects satisfies basic model assumptions. We then discuss the limitations of this approach and the future of identifying markers in the field.

\section{Methods and Analysis}

\section{Background and Operationalization of Theory}

In the Kingdom of Tonga in western Polynesia, native peoples often manufacture a set of motifs or kupesi. Each motif may be found within the kingdom, in neighboring island nations, and in the Tongan diaspora. Since kupesi refers specifically to older designs and the stencil-like materials used by chiefly women to imprint on barkcloth, not all motifs are kupesi. However, all motifs share similar functions. Many of the motifs have names, are taught in Tongan schools, and the manufacture of motifs is a cultural and economic activity tied to important life events in the public sphere. Once reserved for chiefs or nobles in the past, the means of reproduction of kupesi are now common during the exchange of barkcloth, plaited mats, and other items during events such as weddings, funerals, religious eventsm and birthday celebrations. Further, the expression of kupesi appears to be bolstered by transnational ties with expatriate communities across the Pacific Rim. In these Tongan diaspora 
communities, motif presence on T-shirts, cars, and digital media (e.g., websites, personal avatars) gives evidence to their central place in expressions of social identity. As in other parts of the Pacific (Ewins, 2004) the motifs are seen as key representation of social concepts, identity and thus key candidates as signals of group membership. Given these qualities, motifs and kupesi may be categorized as emblemetic style sensu Wiessner (1983) and act as a direct referent to the native ethnolinguistic groups in Tonga and other surrounding islands.

However, as mathematical models assume and highlight above, mechanisms validating a motif as an ethnic marker rely on them being present and observable, widely recognized by individuals, and having information content. We operationalize these features in the population-specific context of Tongan motifs. In this case, being present and observable means they are displayed in prominent social spaces such as public thoroughfares and markets on any type of medium. This stems from the fact that the cultural reproduction of motifs and kupesi is a statement of meeting social obligations, such that their public display is part of the motivation for and continuation of their production.

The second is to assess their recognition among individuals. In the context of Tongan motifs, recognition is operationalized as awareness and knowledge of the motifs. This is distinct from the first condition in that public display of a motif does not mean individuals are aware of the motif as a discrete entity. Consider the common confession that once one learns of something new, then suddenly it is recognized as commonplace. The theory behind covert signaling uses this fact (Smaldino et al., 2018). In the Tongan and broader Pacific Islander context there are numerous motifs, from both pre- and post-European contact, but it does not mean the targeted population is aware and knows of them. After our collection of motifs from the first step, a rapid ethnographic survey was conducted to address the two concepts of awareness and knowledge of each motif for each informant.

Finally, the third related feature is that the motifs have information content. We translate this condition to mean that the target population has been socialized about the motifs in a culturally specific manner. To address the conscious and unconscious elements to marking and information content, we use a comparative classification task to measure whether a set of objects contains information resulting from population-specific socialization. This approach is introduced in detail below.

The three measurement activities were conducted in the following order. We conducted analyses in the statistical program R (R Core Team, 2020), and all code and relevant data files are found on the Github repository avbell/MotifsBellPaegle.

\section{Presence}

Along main public thoroughfares in 2016 in the Tongan capital city of Nuku'alofa and in the Ha' apai island group we conducted a continuous scan (Hames \& Paolisso, 2014) of personal adornments, printed signs, architecture, handicrafts, and any visible medium where kupesi may be expressed. The scans lasted for several hours and yielded 15 motifs originally captured as still pictures and then drawn on a digital tablet (Fig. 1). We included motifs that we knew a priori would be considered kupesi 

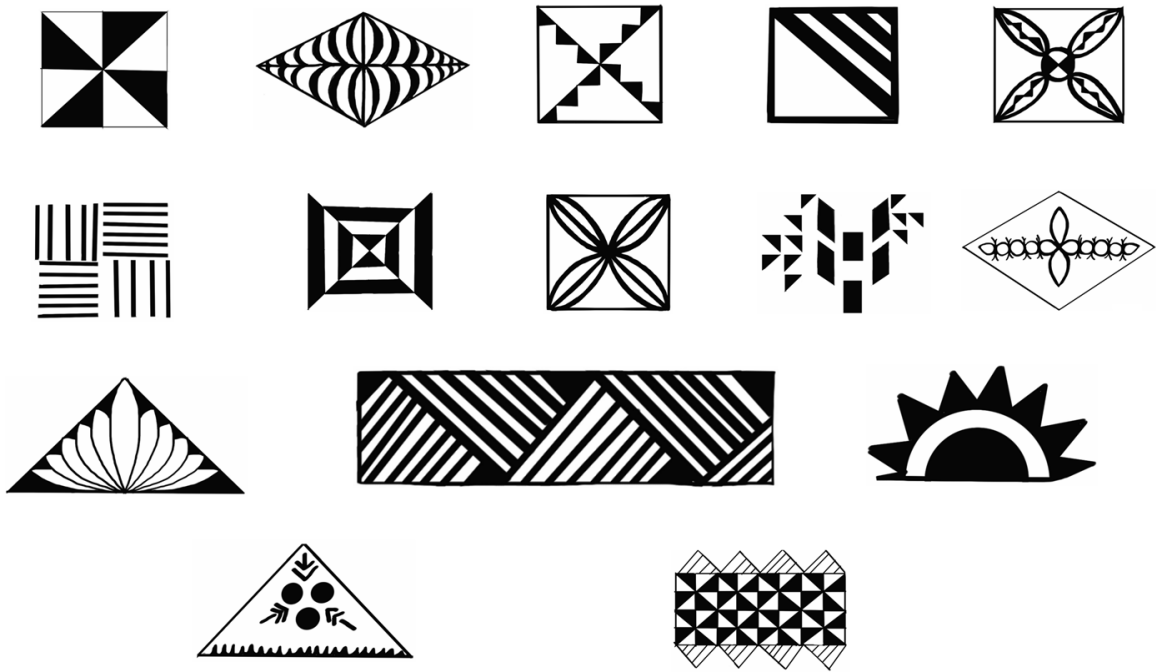

Fig. 1 Fifteen motifs or kupesi gathered from continual scans in public spaces across Nuku'alofa, Tonga, in 2016

by the Tongan population, and we excluded motifs not part of the kupesi category, such as commercial logos and Christian religious motifs. Kupesi observed only once were included in the study. The objective was not to acquire an exhaustive list of motifs known by the population but rather motifs expressed in public at this time.

A number of the kupesi in Fig. 1 have well-documented meanings. For example, the manulua on the very top left is thought to represent the intersection of two winged animals, whose larger significance may mark the joining of chiefly lineages. This is a local variant of a core design of manulua found with some antiquity on Lapita pottery from Melanesia (Cowling, 2009). Immediately to the right of this manulua motif is tokelau feletoa (object 1); although attributed to the northern island group of Vava'u within Tonga, it is seen throughout the Tongan archipelago. The form of tokelau feletoa has multiple interpretations (Arbeit, 1994; Helu, 1999), but they all refer to chiefly actions or representations. Finally, the long rectangular motif with cross-hatched bars is another motif with some antiquity. Called amoamokofe or "inner plant bark with bamboo," the straight line designs reflect the older Tongan design tradition of high abstraction, angularity, and extreme simplicity (Helu, 1999). This motif is considered a key example of Tongan sociospatial relations, or $t \bar{a}-v \bar{a}$ (time-space)philosophy, among indigenous scholars (Mahina, 2004). Not all the motifs in Fig. 1 have such well-documented histories or commentary.

For object-facilitated social coordination to work, the second prerequisite is that objects are broadly recognized. We turn to this question next.

\section{Recognition}

To assess motif and kupesi recognition in Tonga, 70 rapid anonymous surveys were conducted in the same 2016 field season after the collection of the motifs. We 
presented to each participant all 15 motifs on a digital tablet. For each motif we asked (in Tongan) whether they have seen the motif, resulting in a "yes" or "no." If they answered "yes" then they were asked two additional questions: if they knew the name of the motif, resulting in a "no" or a name given, and where they have seen it, usually yielding a response naming one or two mediums (e.g., barkcloth, plaited mats). We recruited participants primarily through street contacting and short house visits. Through this means the sample included a range from 18 to 86 years of age, with 25 participants in the 18-30, 22 in the 31-50, and 23 in the 50+ age classes.

The data from this survey show substantial variation in kupesi recognition. In Fig. 2 the extent of recognition shows a gradual decline across kupesi, with one fairly less well known motif. Further, the naming of specific motifs fell after the first two kupesi on the horizontal axis. While about a third of all participants could name the first two motifs, introduced above as tokelau feletoa and manulua, very few if any could provide a name for the others.

Social information. The reproduction of a marker is contingent on individuals comparing the payoffs of a specific marker and associated behaviors with the average payoffs of others in the population (McElreath et al., 2003). Under conditions in which markers are favored to form, marker-behavior combinations with greater payoffs will increase in frequency. In ethnographic parlance, a marker becomes a salient object of semiotic study and thus carefully organized in relation to other objects in the population. We view the cognitive processes behind this organization ranging from conscious, deliberate, and active to passive and without conscious intent. It is the latter features of passive marking that limit the inferences from direct questioning of informants. Although such instruments may be developed for some cultural

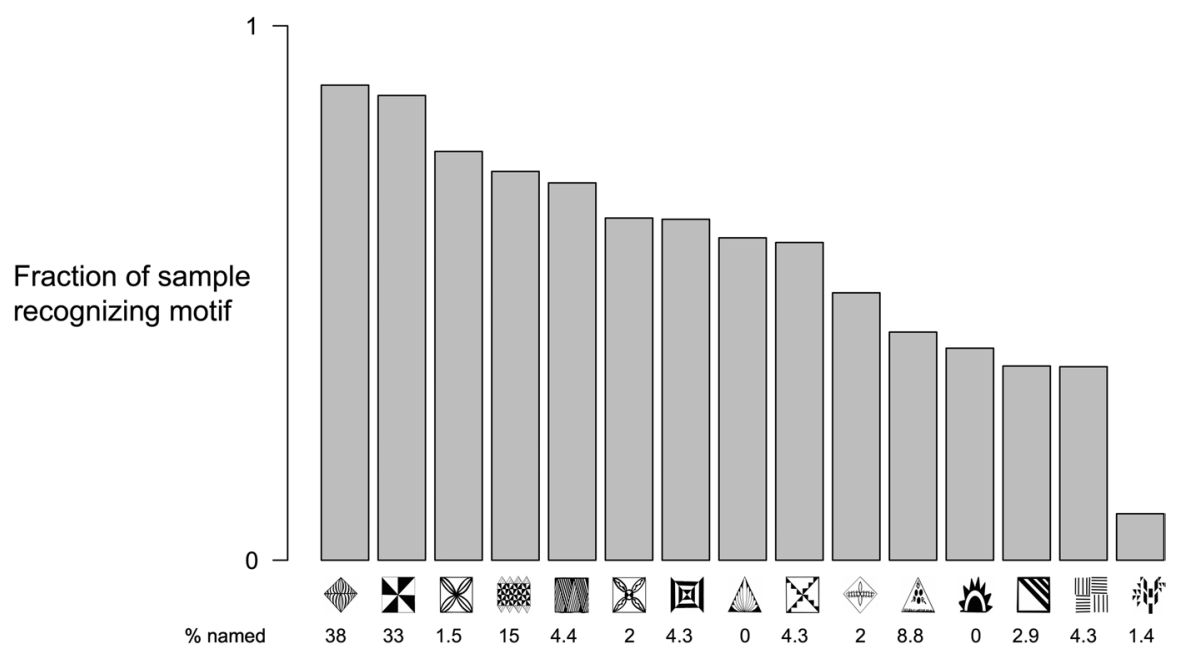

Fig. 2 Recognition of motifs. Motifs along the horizontal axis were the result of a continuous scan in public spaces (Fig. 1). Bars show the fraction of the time the respondents answered "yes" to having seen a motif, and the numbers below the images report the percent of participants providing a name for a motif. Calculations stem from 70 ethnographic surveys in Tonga 
domains, we felt an ethnographic instrument with conscious and unconscious elements would be a better measure of information found in Tongan motifs.

The instrument used here is a classification task. As formally shown in a related paper (Bell, 2020), there is a close relationship between a marker's role in social coordination and its classification among other objects. As a well-established ethnographic method, classification studies help us understand how populations organize what they observe and often how their environment affects that organization (Weller, 2015).

This suggests that there is an indirect method by which to evaluate whether a set of markers contains social information. If a cultural domain plays an important coordinating role, that role is reflected in the way by which a cultural domain is organized within a group, as well as notable classification differences between groups. That is, between-group classification differences can indirectly measure the importance of a set of objects within a group. This idea motivates the next empirical section of this study, a comparative classification study of motifs with Tongan participants in Tonga and a non-Tongan U.S. student sample.

Using the six most recognized motifs among the 15 collected in 2016 (Fig. 2), we used triad tasks in 2018 as a method to reveal pair-wise associations among motifs for each individual. The triad task was conducted on a touch-screen tablet using an HTML interface coded in R Shiny. After viewing an instructional screen and answering questions about age and gender, participants were presented with three motifs and asked to choose the object that is most different by pressing on that object. For six motifs, a possible combination of 20 total triad decisions were made by each participant. Through street contacting and short house visits, 114 participants completed the 5-min anonymous task, with the sample consisting of 55 male and 56 female participants with an age range from 18 to 70 years old. Three participants did not indicate gender. In the U.S., a reference sample of 51 non-Tongan university students participated in the English version of the task.

For each pair of participants we computed the fraction of choice agreement across the Tongan and U.S. sample. That is, for $m$ number of motifs in the set $S$ and for object triad set $K$, the fraction of choice agreement between two individuals was computed as

$$
a_{i, j}=\frac{\sum_{k \in K} I_{i, j}^{k}}{\left(\begin{array}{c}
m \\
3
\end{array}\right)}
$$

where $I_{i, j}^{k}=1$ if individuals $i$ and $j$ made the same choice for object triad $k$, and 0 otherwise. Using these values, we investigated the within- and between-group classification differences via a difference matrix, visualized here with multidimensional scaling and dendrograms (Fig. 3). The plots show both a group of U.S. participants that overlap the Tongan sample and a cluster set apart from the Tongan sample.

The dendrograms in Fig. 3 point to the primary classification differences between the Tongan respondents and the U.S. reference sample. In both groups, flower-like motifs are grouped together. However, the associations made with the kupesi named manulua (top left motif of Fig. 1) and tokelau feletoa (to the right of manulua in 


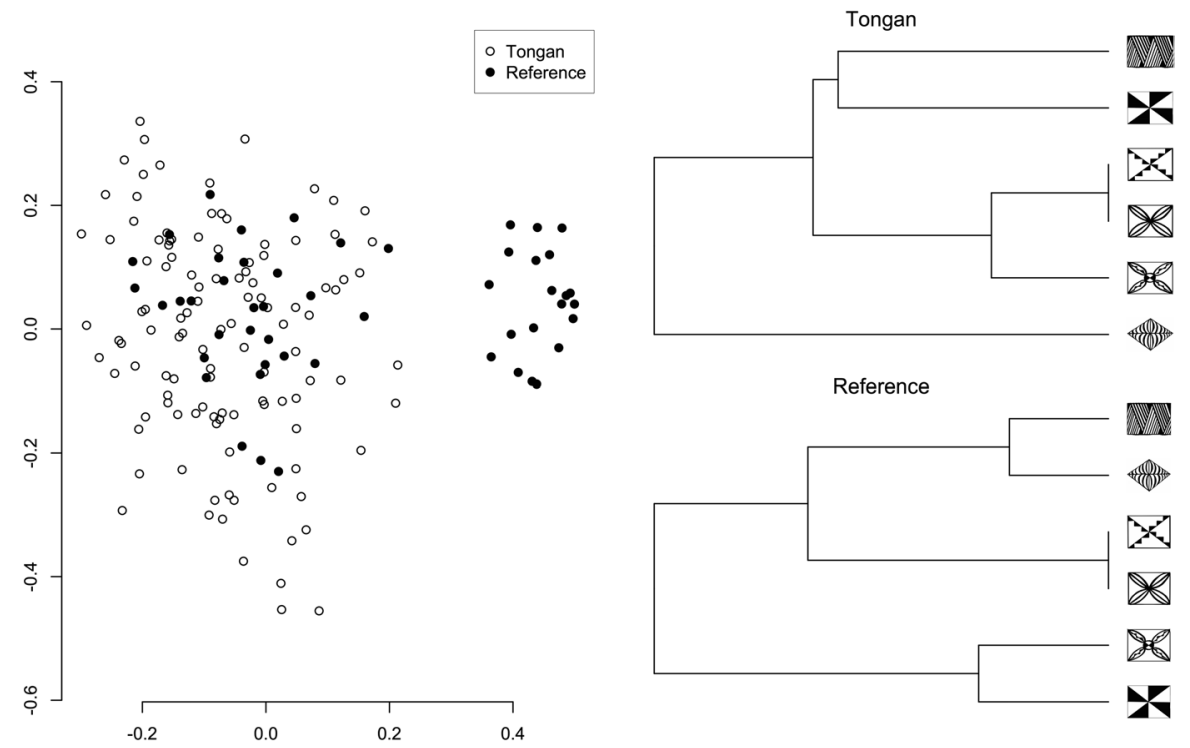

Fig. 3 Similarity of choices in the motif classification task across a Tongan and a reference population. The left panel plots the results of multidimensional scaling, performed on a difference matrix of values across all possible dyads for 114 Tongan participants and 51 U.S. non-Tongan students. Cluster analysis suggests two clusters, with the group of solid points to the right forming a separate cluster from the rest of the plot. The right panel shows dendrograms generated from the same difference matrix, showing where the differences in classification lie between the Tongan and reference sample

Fig. 1) are striking. Tokelau feletoa is grouped apart from the rest of the motifs in the Tongan sample, whereas it is placed with amoamokofe in the reference sample. Manulua is also placed differently between the two populations. Note that these two kupesi are the most well-known in Tonga, witnessed through observation and also by the evidence visualized in Fig. 2. Thus it is not a surprise to see manulua and tokelau feletoa take a distinctive place among motifs due to their cultural importance in Tongan society.

\section{Discussion}

Our ethnographic approach revealed a number of motifs that likely operate as ethnic markers among the Tongan people. Our conclusions came by assessing three prerequisites outlined by theoretical models-presence, recognition, and social informationvia an ethnographic method consisting of scans in public spaces, an ethnographic survey, and a classification task. Scans produced a set of publicly expressed motifs (Fig. 1). A subsequent survey measured each motif's exposure or recognition by the population, with most motifs acknowledged by at least half the sample (Fig. 2). Finally, a comparative classification study with a reference population yielded enough differences to suggest the motifs may be embedded with social information in the target population 
(Fig. 3). While these findings suggest our approach is promising, there are outcomes or limitations that provide guides for improvement.

First, although the study suggests the set of motifs as a whole operate as markers, it does not measure any differences in social importance among motifs. In fact, the ethnographic surveys measuring recognition suggest significant heterogeneity. In hindsight, variability is expected given the history of kupesi in Tonga. Before the changes that occurred after European contact, the reproduction of motifs was directed under the supervision of the chiefly class. The barkcloth were made by commoner women in groups called kautaha, but the motifs and the boards used to imprint on barkcloth were held by chiefly women. This changed during the late nineteenth and early twentieth centuries as chiefly women gradually withdrew from kautaha groups, after which commoner women began to create their own designs (Tamahori, 1963).

Related to this outcome is the quick drop in naming despite recognition (Fig. 2). This is likely influenced by two factors. Not all motifs are kupesi with well-documented names or a known history. Further, while naming may be important in the social discussion of kupesi, naming is not required for motifs to function as ethnic markers. What is required by theory is that individuals recognize not only each motif but also how the motifs relate to each other in a social setting. This suggests that measuring the role of each motif would require data and statistical methods linking the value of each motif to the associations among motifs. This idea is given a formal treatment elsewhere (Bell, 2020).

This paper reports a classification task as an instrument to measure the amount of culture-specific socialization on a motif set. While this approach addresses active/ conscious and passive/unconscious cognitive processes that are involved in group marking, other instruments may be developed to measure the information content of candidate markers. For example, emic narratives about markers may specify the specific roles of kupesi and nominate certain design elements. Prompting informants to identify the group affiliation of objects or individuals may isolate the role of specific features (e.g., Bowser, 2000), though we believe greater statistical development may make better inferences given the high dimensionality of the problem. The combinatorics of motifs along with the context-specific nature of their use present a large task for empiricists. However, we advocate that the instruments involve both conscious and unconscious cognitive processes. At least in the Tongan case, as one of the authors (Bell) has observed, being part of the Tongan community, group marking using motifs is sufficiently complex and dynamic that its discussion among Tongans is articulated and barely uttered, scrutinized and superficially scanned, interpreted and plainly copied.

The temporal dynamics of a set of ethnic markers are not addressed here. New objects are inevitably going to be adopted as migration and the forces of globalization influence homeland and diaspora populations. We already see this in museum ethnographic descriptions and oral histories of Oceanic motifs. The consistency and relationship of motif design across large regions reflect migration history (Kirch, 2000) and the spheres of interaction (Weisler, 1998) that connect Pacific Island populations in the present day. Documented on canoes (Johns et al., 2014) and pottery (Spriggs, 1990) before European contact, their postcontact expression is 
well-described as the focus of symbolic art (Helu, 1999; Kaeppler, 2002; Mahina, 2004). A cross-sectional study of the kind presented here cannot answer questions concerning the life span or life cycle of an ethnic marker that may be afforded by a historical or longitudinal study. This may be essential for studies of social coordination among migrating populations. It may also be needed for the study of tag-based cooperation, as theory requires markers to change through time to avoid exploitation of cooperators by free-riders (Jansen \& Baalen, 2006; Riolo et al., 2001; Traulsen \& Nowak, 2007).

The empirical approach of this paper is highly reliant on the insights from a limited set of theoretical models. However, models are caricatures of reality and therefore cannot capture all the relevant dynamics at play in empirical studies. For example, presumed markers across studies have highly varied features, ranging from the flexible use of clothing to the more permanent tattoos, scarring, and accents. This varied permanence is not in the models. Sociodemographic complexity is also a concern. While McElreath et al. (2003) simulated with multiple groups, the more complex network structures, migration patterns (Bell, 2013), and between-group interactions (Bunce \& McElreath, 2018) will influence individual expression. Covert signaling is not addressed here either (Smaldino et al., 2018). Therefore, the empirical effort here can be seen not as exhausting the conditions for identifying group markers, especially as groups respond dynamically to their environment, but as satisfying basic conditions to build upon.

Relatedly, the results of this work position a study asking whether these motifs facilitate greater social coordination or cooperative outcomes outside of controlled conditions. As emphasized by Ostrom (2010) in a review of polycentric governance, key factors for successful cooperation include communication and finding trustworthy reciprocators. This suggests that external validation of these motifs may include evaluating whether the presence of motifs enables a categorically distinct social interaction-in other words, more communication and more trust.

Acknowledgments Communities in the Kingdom of Tonga graciously participated in field data collection, including local research assistants. Student field assistants Swayze Hansen, Mairanda Henline, and Brittany Kiser were also vital to data collection. Fieldwork was funded by the Max Planck Institute for Evolutionary Anthropology and the University of Utah. We thank anonymous reviewers for their constructive comments.

\section{Declarations}

Ethical Approval All ethnographic activities were approved beforehand by the University of Utah IRB, study \#00,050,947.

Conflict of Interest The authors declare that they have no conflict of interest.

\section{References}

Arbeit, W. (1994). Tapa in Tonga. University of Hawaii Press.

Axelrod, R., \& Hamilton, W. D. (1981). The evolution of cooperation. Science, 211(4489), 1390-1396. 
Bell, A. V. (2013). The dynamics of culture lost and conserved: demic migration as a force in new diaspora communities. Evolution and Human Behavior, 34(1), 23-28.

Bell, A. V. (2020). A measure of social coordination and group signaling in the wild. Evolutionary Human Sciences, 2, e34.

Bowser, B. J. (2000). From pottery to politics: an ethnoarchaeological study of political factionalism, ethnicity, and domestic pottery style in the Ecuadorian Amazon. Journal of Archaeological Method and Theory, 7(3), 219-248.

Boyd, R., \& Richerson, P. J. (1987). The evolution of ethnic markers. Cultural Anthropology, 2(1), 65-79.

Bunce, J., \& McElreath, R. (2018). Sustainability of minority culture when inter-ethnic interaction is profitable. Nature Human Behaviour, 2(3), 205-212.

Cohen, E. (2012). The evolution of tag-based cooperation in humans. Current Anthropology, 53(5), 558-616.

Cohen, E., \& Haun, D. (2013). The development of tag-based cooperation via a socially acquired trait. Evolution and Human Behavior, 34(3), 230-235.

Cowling, W. E. (2009). The lapita motif that got away. Sites: A Journal of Social Anthropology and Cultural Studies, 6(2):57-79.

Efferson, C., Lalive, R., \& Fehr, E. (2008). The coevolution of cultural groups and ingroup favoritism. Science, 321(5897), 1844-1849.

Ewins, R. (2004). Symmetry and semiotics: The case of Fijian barkcloth figuration. In D. Washburn (Ed.), Embedded symmetries, natural and cultural (pp. 161-183). Amerind New World Studies Series. University of New Mexico Press.

Hames, R., and Paolisso, M. (2014). Behavioral observation. In H. R. Bernard and C. C. Gravlee (Eds.), Handbook of methods in cultural anthropology (2nd edition, pp. 293-312). Rowman \& Littlefield.

Hamilton, W. D. (1964). The genetical evolution of social behavior, parts I and II. Journal of Theoretical Biology, 7(1-16), 17-52.

Helu, F. (1999). Critical essays: Cultural perspectives from the South Seas. Canberra: Journal of Pacific History.

Hodder, I. (1977). The distribution of material culture items in the Baringo district, western Kenya. Man, 239-269.

Jansen, V. A. A., \& Baalen, M. V. (2006). Altruism through Beard chromodynamics. Nature, 440(7084), 663-666.

Johns, D. A., Irwin, G. J., \& Sung, Y. K. (2014). An early sophisticated east Polynesian voyaging canoe discovered on New Zealand's coast. Proceedings of the National Academy of Sciences, 111(41), $14728-14733$.

Kaeppler, A. L. (2002). The structure of Tongan barkcloth design: Imagery, metaphor and allusion. In Anita Herle (Ed.), Pacific art: Persistence, change, and meaning (pp. 291-308). University of Hawai'i Press.

Kirch, P. V. (2000). On the road of the winds: an archeological history of the Pacific Islands before European contact. University of California Press.

Mahina, O. (2004). Art as tā-vā time-space transformation. University of Auckland.

McElreath, R., Boyd, R., \& Richerson, P. J. (2003). Shared norms and the evolution of ethnic markers. Current Anthropology, 44(1), 122-129.

McPherson, M., Smith-Lovin, L., \& Cook, J. M. (2001). Birds of a feather: Homophily in social networks. Annual Review of Sociology, 27(1), 415-444.

Moya, C., \& Boyd, R. (2016). The evolution and development of inferential reasoning about ethnic markers: comparisons between urban United States and rural highland Peru. Current Anthropology, 57(S13), S131-S144.

Ostrom, E. (2010). Beyond markets and states: Polycentric governance of complex economic systems. American Economic Review, 100(3), 641-672.

$\mathrm{R}$ Core Team (2020). R: A language and environment for statistical computing. R Foundation for Statistical Computing, Vienna, Austria.

Riolo, R. L., Cohen, M. D., \& Axelrod, R. (2001). Evolution of cooperation without reciprocity. Nature, 414(6862), 441-443.

Smaldino, P. E., Flamson, T. J., \& McElreath, R. (2018). The evolution of covert signaling. Scientific Reports, 8(1), 4905.

Spriggs, M. (1990). The changing face of Lapita: Transformation of a design. In Lapita design, form and composition. Proceedings of the Lapita Design Workshop, Canberra, Australia December 
1988 (pp. 83- 122). Department of Prehistory, Research School of Pacific Studies, Australian National University.

Tamahori, M. J. (1963). Cultural change in Tongan bark-cloth manufacture. PhD thesis, University of Auckland.

Traulsen, A., \& Nowak, M. A. (2007). Chromodynamics of cooperation in finite populations. PLoS ONE, 2(3), e 270.

Tsuda, T. (2009). Diasporic homecomings: Ethnic return migration in comparative perspective. Stanford University Press.

Weisler, M. I. (1998). Hard evidence for prehistoric interaction in Polynesia. Current Anthropology, 39(4), 521-532.

Weller, S. C. (2015). Structured interviewing and questionnaire construction In H. R. Bernard \& C. C. Gravlee (Eds.), Handbook of methods in cultural anthropology (2nd ed., pp. 343-390): Rowman \& Littlefield.

Wiessner, P. (1983). Style and social information in Kalahari San projectile points. American Antiquity, 253-276.

Wiessner, P. (1984). Reconsidering the behavioral basis for style: A case study among the Kalahari San. Journal of Anthropological Archaeology, 3(3), 190-234.

Wimmer, A., \& Lewis, K. (2010). Beyond and below racial homophily: ERG models of a friendship network documented on Facebook. American Journal of Sociology, 116(2), 583-642.

Publisher's Note Springer Nature remains neutral with regard to jurisdictional claims in published maps and institutional affiliations.

Adrian Viliami Bell (PhD Human Ecology 2011, UC Davis) is associate professor of anthropology at the University of Utah. He is primarily interested in the effect of migration on cultural evolution and uses mathematical models alongside active fieldwork to answer fundamental questions about the human condition.

Alina Paegle (BS Anthropology 2018, University of Utah) is currently a graduate student pursuing a Master's of Science in Public Health (MSPH) at the University of Utah School of Medicine, Division of Public Health. She is also an epidemiologist at the Utah Department of Health who is actively assisting in the COVID-19 pandemic response. Her interests range from infectious disease epidemiology and medical microbiology, to human ecology and human evolutionary biology. 